\title{
Loss Mechanisms and Quasiparticle Dynamics in Superconducting Microwave Resonators Made of Thin-Film Granular Aluminum
}

\author{
Lukas Grünhaupt, ${ }^{1}$ Nataliya Maleeva, ${ }_{1}^{1}$ Sebastian T. Skacel, ${ }^{1}$ Martino Calvo, ${ }^{2}$ Florence Levy-Bertrand, ${ }^{2}$ \\ Alexey V. Ustinov, ${ }^{1,3}$ Hannes Rotzinger, ${ }^{1}$ Alessandro Monfardini, ${ }^{2}$ Gianluigi Catelani, ${ }^{4}$ and Ioan M. Pop ${ }^{1,5,}$ \\ ${ }^{1}$ Physikalisches Institut, Karlsruhe Institute of Technology, 76131 Karlsruhe, Germany \\ ${ }^{2}$ Université Grenoble Alpes, CNRS, Grenoble INP, Insitut Néel, F-38000 Grenoble, France \\ ${ }^{3}$ Russian Quantum Center, National University of Science and Technology MISIS, 119049 Moscow, Russia \\ ${ }^{4}$ JARA Institute for Quantum Information (PGI-11), Forschungszentrum Jülich, 52425 Jülich, Germany \\ ${ }^{5}$ Institute of Nanotechnology, Karlsruhe Institute of Technology, 76344 Eggenstein Leopoldshafen, Germany
}

(Received 9 February 2018; published 11 September 2018)

\begin{abstract}
Superconducting high kinetic inductance elements constitute a valuable resource for quantum circuit design and millimeter-wave detection. Granular aluminum ( $\mathrm{grAl}$ ) in the superconducting regime is a particularly interesting material since it has already shown a kinetic inductance in the range of $\mathrm{nH} / \square$ and its deposition is compatible with conventional $\mathrm{Al} / \mathrm{AlOx} / \mathrm{Al}$ Josephson junction fabrication. We characterize microwave resonators fabricated from grAl with a room temperature resistivity of $4 \times 10^{3} \mu \Omega \mathrm{cm}$, which is a factor of 3 below the superconductor to insulator transition, showing a kinetic inductance fraction close to unity. The measured internal quality factors are on the order of $Q_{i}=10^{5}$ in the single photon regime, and we demonstrate that nonequilibrium quasiparticles (QPs) constitute the dominant loss mechanism. We extract QP relaxation times in the range of $1 \mathrm{~s}$ and we observe QP bursts every $\sim 20 \mathrm{~s}$. The current level of coherence of grAl resonators makes them attractive for integration in quantum devices, while it also evidences the need to reduce the density of nonequilibrium QPs.
\end{abstract}

DOI: 10.1103/PhysRevLett.121.117001

Superconducting materials with a high kinetic inductance play a prominent role in superconducting circuits, such as quantum bits (qubits) with remarkably high energy relaxation times [1-4], topological [5,6] and protected qubits [7-9], coherent quantum phase slip circuits [10-13], wideband parametric amplifiers $[14,15]$, and resonators for quantum state of light engineering $[16,17]$. As the kinetic inductance fraction $\alpha=L_{\text {kinetic }} / L_{\text {total }}$ increases, so does the susceptibility of superconducting circuits to quasiparticle (QP) excitations, which constitutes an asset for kinetic inductance detectors (KIDs) [18]. In contrast, for quantum information, circuits are heavily shielded, in an effort to minimize the generation of excess QPs, due to photons, phonons, or other particles with energies larger than twice the superconducting gap. Even residual QP densities as low as $10^{-6}$, normalized to the density of Cooper pairs, can be responsible for excess decoherence in superconducting quantum circuits [2,19-24]. For temperatures much lower than the critical temperature, in the limit of weak microwave drive, the origin and dynamics of excess QPs is an active field of research [22,23,25-29], with direct implications for quantum computation with Majorana modes [30,31].

In this Letter we report the measurement of QP dynamics in microwave resonators fabricated from superconducting granular aluminum (grAl) [32-35], with a kinetic inductance fraction close to unity [36]. We observe QP bursts every $\sim 20 \mathrm{~s}$ and steady-state $\mathrm{QP}$ relaxation times $\tau_{\mathrm{ss}}$ in the range of $1 \mathrm{~s}$ at a temperature $\sim 25 \mathrm{mK}$. Despite such remarkably long relaxation times, we show that grAl resonators with a kinetic inductance $L_{\text {kinetic }}$ as high as $2 \mathrm{nH} / \square$ maintain internal quality factors $Q_{i}>10^{5}$ for $\bar{n} \approx 1$ average circulating photons. These properties place grAl in the same class of low-loss, high kinetic inductance environments as Josephson junction arrays [37-39] and disordered superconducting thin films, such as TiN [40-42], NbTiN [43-45], and $\mathrm{NbN}$ [46-48].

Granular aluminum films owe their name to the self-assembly of pure aluminum grains, $\sim 3 \mathrm{~nm}$ in diameter $[33,35]$, inside an aluminum oxide matrix, during the deposition of pure aluminum in an oxygen atmosphere ( $p_{\mathrm{Ox}} \sim 10^{-5} \mathrm{mbar}$ ). Controlling the oxygen pressure allows the fabrication of films with resistivities $\rho$ in the range of $1-10^{5} \mu \Omega \mathrm{cm}$. The main results in this Letter are obtained for resonators (see Fig. 1) fabricated from a grAl film with $\rho=4 \times 10^{3} \mu \Omega \mathrm{cm}$, a factor of 3 below the superconductor to insulator transition (SIT) $[32,49]$, and a corresponding $T_{c}=2.1 \mathrm{~K}$. The resistivity was chosen as high as possible, to maximize the kinetic inductance $L_{\text {kinetic }} \propto \rho$ [36], while remaining sufficiently below the SIT, where quantum fluctuations and film inhomogeniety start dominating the microwave properties [50].

Figure 1 shows a photograph of a 3D copper waveguide sample holder, which provides a low loss microwave environment and reduces the surface dielectric participation 


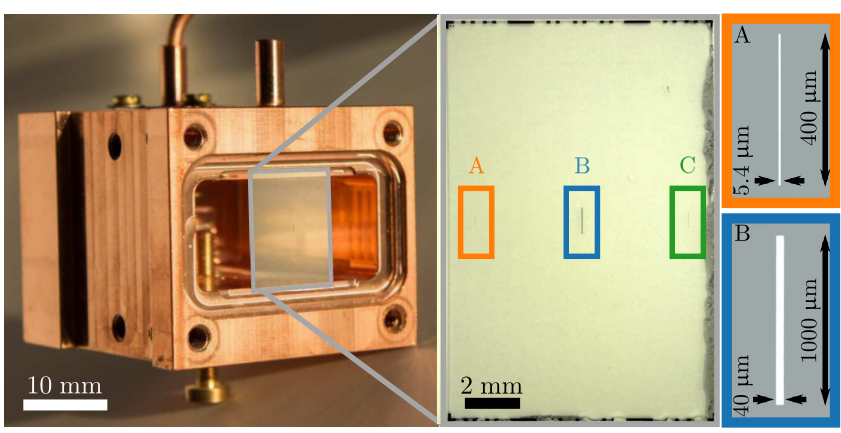

FIG. 1. Optical images of the grAl resonators and the 3D waveguide sample holder. The copper waveguide provides a low-loss environment [51,52] for three grAl resonators patterned by $e$-beam lift-off lithography on a $10 \times 15 \mathrm{~mm}^{2} c$-plane sapphire chip. The resonator dimensions and resonant frequencies $f$ are the following: $A-400 \times 5.4 \mu \mathrm{m}^{2}, f=6.994 \mathrm{GHz}$; $B-1000 \times 40 \mu \mathrm{m}^{2}, f=6.025 \mathrm{GHz}$; and $C-600 \times 10 \mu \mathrm{m}^{2}$, $f=6.287 \mathrm{GHz}$. The $20 \mathrm{~nm}$ thick grAl film has a resistivity $\rho=4 \times 10^{3} \mu \Omega \mathrm{cm}$, corresponding to a sheet resistance $R_{s}=$ $2 \mathrm{k} \Omega / \square$. By comparing the measured resonant frequencies with a finite elements method (FEM) simulation we extract a kinetic inductance $L_{\text {kinetic }}=2 \mathrm{nH} / \square$.

ratio [51,52]. The $20 \mathrm{~nm}$ thick grAl resonators are patterned on a $10 \times 15 \mathrm{~mm}^{2} c$-plane sapphire chip, using $e$-beam lift-off lithography, on a PMMA/MMA bilayer. By varying the length of the resonators, we tune the coupling quality factor $Q_{c}$ from $4 \times 10^{3}$ to $6 \times 10^{4}$ (cf. Fig 1 ).
We perform standard microwave reflection measurements in commercial dilution cryostats at a base temperature $T \approx 25 \mathrm{mK}$. The waveguide sample holders are successively surrounded by a series of shields and absorbing materials, to minimize stray radiation and magnetic fields (cf. [53] and Supplemental Material [54]).

Comparing the measured resonant frequencies of the grAl resonators with FEM simulations (see Supplemental Material [54]), we infer a kinetic inductance $L_{\text {kinetic }}=$ $2 \mathrm{nH} / \square$ for $\rho=4 \times 10^{3} \mu \Omega \mathrm{cm}$. The corresponding kinetic inductance fraction $\alpha$ is expected to be close to unity, as confirmed by measurements in Fig. 2(a). For superconducting quantum circuits with $\alpha=1$, internal quality factors $Q_{i}$ could start to be limited by residual QPs. We measure single photon $Q_{i}$ on the order of $10^{5}$ [see Fig. 2(b)], comparable with other realizations of high kinetic inductance materials $[38,40,44,58]$, which could be explained by a residual excess quasiparticle density $x_{\mathrm{QP}}=5 \times 10^{-6}$, in the range of previously reported values [2,19-24].

Figure 2(b) also shows the $Q_{i}$ dependence on the average circulating photon number $\bar{n}=4 P_{\text {in }} Q_{\text {tot }}^{2} /\left(\hbar \omega_{r}^{2} Q_{c}\right)$ [62], here $P_{\text {in }}$ is estimated from the output power of the vector network analyzer by taking into account the total attenuation in the cryostat (see Supplemental Material [54]). For resonator $A, Q_{i}$ shows an increase by a factor of 4 between $\bar{n}=1$ and 100 , reaching $6 \times 10^{5}$ before the resonator bifurcates due to its intrinsic nonlinearity [63]. Seven times larger in cross section, resonator $B$ shows a smaller increase


FIG. 2. Measurement of radio frequency loss mechanisms in grAl films. (a) Measurement of the relative shift of the resonant frequency $\delta f / f=[f(T)-f(0.02)] / f(0.02)$ as a function of temperature. From FEM simulations we expect the kinetic inductance to be three orders of magnitude larger than the geometric inductance. The data can be fitted using a Bardeen-Cooper-Schrieffer (BCS) model [59,60], $\delta f(T) / f=-\frac{\alpha}{2} \sqrt{\pi \Delta_{0} /\left(2 k_{B} T\right)} \exp \left(-\Delta_{0} / k_{B} T\right)$, which is expected to approximately describe the temperature dependence of the frequency, but which does not take into account corrections to the prefactor which can arise either as temperature changes from below to above $h f / k_{B}$ or due to deviations of grAl from standard BCS theory [32]. The black line shows the fit for the kinetic inductance fraction $\alpha=1$. We extract a value for the grAl superconducting gap $\Delta_{0}=(288 \pm 4) \mu \mathrm{eV}$, in agreement, within $15 \%$, with the measured gap from THz spectroscopy [32,49], and previously reported values [33]. Notice that the grAl gap is $\sim 1.6$ times larger than that of thin film aluminum. When using $\alpha$ as a free fit parameter, we obtain similar values $\left(\alpha=0.92 \pm 0.06, \Delta_{0}=(282 \pm 4) \mu \mathrm{eV}\right)$. (b) Measured internal quality factors $Q_{i}$ as a function of the average circulating photon number $\bar{n}$. The solid lines represent a fit to the QP activation model of Eq. (1), discussed in the main text. (c) Comparison between measured $Q_{i}$ in the single photon regime as a function of the metal-substrate participation ratio $p_{\mathrm{MS}}$ for different resonator geometries (see Supplemental Material [54]). These results suggest that high kinetic inductance grAl resonators $A-C$ are limited by excess QPs, not by surface dielectric loss. grAl resonators in CPW geometry, with $p_{\mathrm{MS}}>10^{-3}$, are limited by a surface dielectric loss tangent $\tan (\delta)=2.4 \times 10^{-3}$, similar to aluminum qubits [61]. For a detailed discussion see the main text. 
in $Q_{i}$, which is less than a factor of 2 for $\bar{n}$ between 1 and $10^{4}$. The internal quality factor of resonator $C$ could not be fitted above $\bar{n} \approx 1$ because the amplitude data show irregular behavior, changing from the expected dip to a peak (see Supplemental Material [54]).

The measured increase of $Q_{i}$ with $\bar{n}$ can be attributed to the saturation of dielectric loss $[64,65]$, or the activation of QPs [22,27]. The measurements summarized in Fig. 2(c) offer additional insight into the dominant loss mechanism for resonators $A-C$. They indicate that $Q_{i}$ is $\mathrm{QP}$ limited, and consequently suggest a small contribution from dielectric loss saturation to the $Q_{i}$ power dependence.

Concretely, Fig. 2(c) shows a comparison of measured single photon $Q_{i}$ for different grAl resonator geometries as a function of their metal-substrate interface participation ratio $p_{\mathrm{MS}}$, defined as the ratio between the energy stored in the $3 \mathrm{~nm}$ thick interface between the metal and the substrate and the total energy of the mode, following the methodology in Refs. [61,66,67]. The results for resonators $A-C$, shown by triangles and a circle, are about a factor of 10 lower than expected from the typically measured dielectric loss tangent $\tan (\delta)=2.6 \times 10^{-3}$ [61], indicated by the purple dashed line $Q_{i}=\left[p_{\mathrm{MS}} \tan (\delta)\right]^{-1}$. This could either be explained by a 10 times larger dielectric loss tangent associated with the grAl film, indicated by the red dashed line, or by the presence of an additional loss mechanism, such as QP dissipation, independent of $p_{\mathrm{MS}}$ (see grey dashed line).

In order to distinguish between these two possibilities, we measured single photon $Q_{i}$ for grAl samples with increasingly larger $p_{\mathrm{MS}}$. To perform these control experiments, we employed coplanar waveguide (CPW) resonator designs. The blue pentagons in Fig. 2(c) show that increasing the $p_{\mathrm{MS}}$ by a factor of 10 does not degrade the quality factor. To observe a decrease in $Q_{i}$ by a factor of 5 , we had to increase the $p_{\mathrm{MS}}$ by 2 orders of magnitude compared to samples $A-C$, indicating that dielectric loss is not dominant. Surprisingly, when the ground plane is fabricated from aluminum, we observe an increase of $Q_{i}$ by a factor of 2 for an increase of the $p_{\mathrm{MS}}$ by a factor of 10 , presumably due to phonon trapping in the lower gap aluminum ground plane [68]. This result directly suggests QPs are a dominant dissipation source, which is confirmed by measurements on aluminum resonators [53], with 10 times smaller $\alpha$ compared to resonators $A-C$, and similar $p_{\mathrm{MS}}$, showing approximately a factor of ten increase in $Q_{i}$ [cf. grey rhombus in Fig. 2(c)].

A possible source of excess QPs could be impacts of high energy particles, documented in the KID community [69-71]. By continuously monitoring the phase response of the resonators, we observe sudden drops of the resonant frequency, appearing stochastically every $\sim 20 \mathrm{~s}$ (see Supplemental Material [54]), as shown in Fig. 3(a), followed by a remarkably long relaxation process on the timescale of seconds [cf. Fig. 3(b)]. Following a QP burst, the resulting QP density change, $\delta x_{\mathrm{QP}}(t)$, decreases the number of Cooper pairs, thereby increasing the kinetic inductance and lowering the resonant frequency $f$ by $2 \delta f(t) \propto-f \alpha \delta x_{\mathrm{QP}}(t)[23]$.

Figure 3(c) shows the relaxation of $\delta x_{\mathrm{QP}}(t)$ following a QP burst, obtained from an average over tens of individual events, over the course of $45 \mathrm{~min}$ (see Supplemental Material [54]). Notice that the QP relaxation only becomes exponential at long timescales where it is dominated by single QP relaxation, with characteristic timescale $\tau_{\mathrm{ss}}$, whereas at short times the process is governed by QP
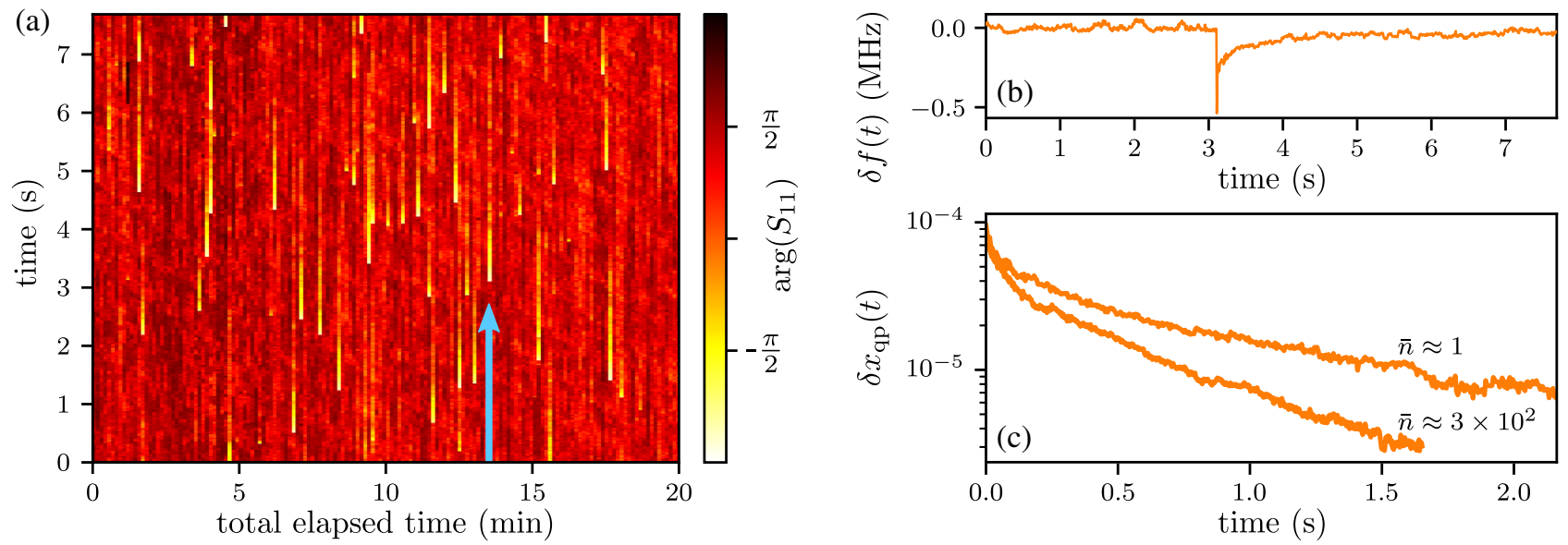

FIG. 3. Measurement of QP generating events. (a) Typical plot of a continuous monitoring of a resonator's phase signal at one frequency point. Multiple time traces are consecutively recorded, covering a total time of about 45 min (for clarity only partially shown). The measurement reveals discrete jumps of the resonant frequency to a lower value followed by a relaxation over seconds approximately every $20 \mathrm{~s}$. (b) Plot of the time trace indicated by the arrow in panel (a), where the phase response is converted into a frequency shift, showing an instantaneous drop followed by a slow relaxation. (c) By recording multiple events and averaging them (see Supplemental Material [54]), an exponential tail can be seen. The characteristic relaxation time, $\tau_{\text {ss }}$, depends on the average circulating photons $\bar{n}$. 
recombination, as previously evidenced in superconducting qubits [23]. Surprisingly, $\tau_{\mathrm{ss}}$ depends on $\bar{n}$, as shown by the two curves in Fig. 3(c), changing from $\tau_{\mathrm{ss}} \approx 0.6 \mathrm{~s}$ at $\bar{n}=300$, to $\tau_{\mathrm{ss}} \approx 1-7 \mathrm{~s}$ in the single photon regime [see Fig. 4(a)]. The statistical error for each $\tau_{\mathrm{ss}}$ measurement is much smaller than the observed scattering between the points, possibly due to slow, $1 / f$ fluctuations in the background QP population [2,72].

The dependence of $\tau_{\mathrm{ss}}$ on $\bar{n}$ suggests that the circulating power in the resonator can accelerate QP diffusion, as reported in qubits [27] and nanobridge junctions [22]. This behavior is different from the previously reported QP generation in aluminum resonators at large driving powers, where $\bar{n} \approx 10^{6}$ [73]. In the presence of disorder, there are spatial variations of the superconducting order parameter where QPs can be localized, giving rise to a small subgap tail in the density of states (DOS) [74]. Similarly to Ref. [75], we develop a phenomenological model that accounts for QP generation, QP recombination, and transition between localized, $x_{l}$, and mobile, $x_{m}$, QP densities:

$$
\begin{aligned}
\dot{x}_{m} & =-\Gamma_{m m} x_{m}^{2}-\Gamma_{m l} x_{m} x_{l}-\Gamma_{\mathrm{loc}} x_{m}+\Gamma_{\mathrm{ex}} x_{l}+g_{m}, \\
\dot{x}_{l} & =-\Gamma_{l l} x_{l}^{2}-\Gamma_{m l} x_{m} x_{l}+\Gamma_{\mathrm{loc}} x_{m}-\Gamma_{\mathrm{ex}} x_{l}+g_{l} .
\end{aligned}
$$

In our model, $\Gamma_{m m}, \Gamma_{l l}$, and $\Gamma_{m l}$ are rates at which two mobile, two localized, or a mobile and a localized QP recombine. $\Gamma_{\mathrm{loc}}$ and $\Gamma_{\mathrm{ex}}$ account for QP localization and
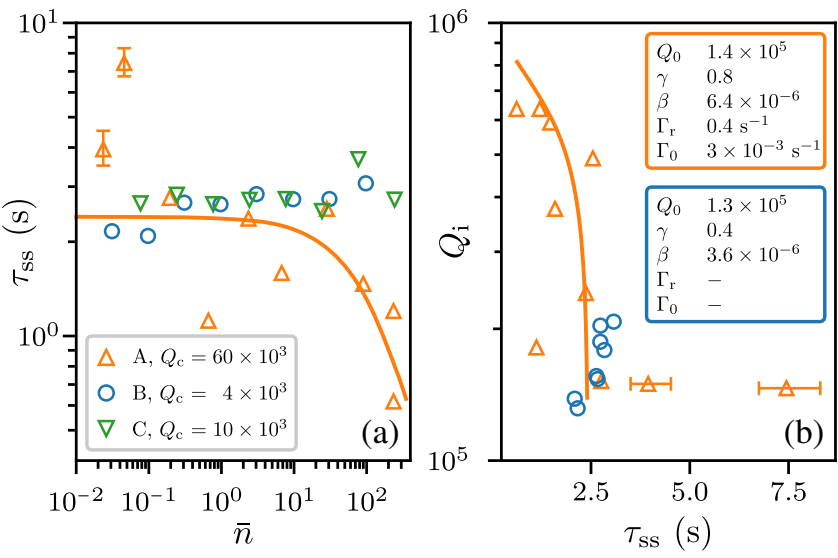

FIG. 4. Steady state QP relaxation constant $\tau_{\mathrm{ss}}$ as a function of $\bar{n}$, and its correlation with $Q_{i}$. (a) Resonator $A$ (orange triangles) shows a decrease of $\tau_{\mathrm{ss}}$ by approximately an order of magnitude whereas $\tau_{\mathrm{ss}}$ of $B$ and $C$ stays constant, within a factor of 2 . The solid line is a fit to the phenomenological model described by Eq. (2). All fit parameters are given in the inset of panel (b). Error bars, where not plotted, are approximately the size of the marker and represent the statistical error of the fit (see Supplemental Material [54]). (b) Correlation between $\tau_{\mathrm{ss}}$ and $Q_{i}$ for resonators $A$ and $B$. We obtain $\tau_{\mathrm{ss}}$ and $Q_{i}$ in separate measurements and plot $Q_{i}\left(\tau_{\mathrm{ss}}\right)$ for values measured at the same photon number. The orange line is traced using the fits for the measured $\tau_{\mathrm{ss}}$ [panel (a)] and $Q_{i}$ [Fig. 2(b)]. excitation (due to photon interaction) from states in the subgap tail of the DOS, while $g_{m}$ and $g_{l}$ describe the generation of mobile and localized QPs. The photon assisted excitation of localized QPs can be modeled by $\Gamma_{\mathrm{ex}}=\Gamma_{0} \bar{n}$, where $\Gamma_{0}$ accounts for the strength of photon interaction with QPs. In principle, both mobile and localized QPs contribute to loss, proportional to their normalized density, coupling strength, and final DOS. Assuming a larger final DOS for localized QPs, and further simplifications (see Supplemental Material [54]), we model the photon number dependent loss due to localized QPs,

$$
\frac{1}{Q_{i}}=\frac{1}{Q_{0}}+\beta\left(\frac{1}{1+\frac{\gamma \bar{n}}{1+\frac{1}{2}(\sqrt{1+4 \gamma \bar{n}}-1)}}-1\right) .
$$

Here, $1 / Q_{0}$ quantifies residual loss mechanisms independent of $\bar{n}, \gamma=2 \Gamma_{\mathrm{loc}} \Gamma_{0} /\left(g_{m} \Gamma_{m l}\right)$, and $\beta \propto \Gamma_{\text {loc }} / \Gamma_{m l}$, but also accounts for QP-photon coupling strength, and the final DOS. Similarly, we model $1 / \tau_{\mathrm{ss}}$ by a residual decay rate $\Gamma_{r}$ and a photon dependent part,

$$
\frac{1}{\tau_{\mathrm{ss}}}=\Gamma_{r}+\Gamma_{0}\left[\bar{n}+\frac{1}{2 \gamma}(\sqrt{1+4 \gamma \bar{n}}-1)\right],
$$

which indicates that QPs relax faster at higher photon numbers $\bar{n}$, when they are activated from localized to mobile.

The fit parameters for resonators $A$ and $B$ are given in the insets in Fig. 4(b). As expected from Fig. 2(b), both resonators show comparable residual quality factors, $Q_{0} \approx Q_{i}(\bar{n} \ll 1)$. The ratio between the $\beta$ coefficients for resonators $A$ and $B$ is $\sim 2$, comparable to the ratio between the $\gamma$ coefficients. Since both $\beta$ and $\gamma$ are proportional to the QP-photon coupling (see Supplemental Material [54]), the factor of 2 difference between resonator $A$ and $B$ might be explained by the reduced current density per photon in resonator $B$, due to its seven times wider cross section. The flat behavior of $\tau_{\mathrm{ss}}$ for resonator $B$ suggests that the QP-photon coupling is not sufficient to excite localized QPs. Consequently, the small increase in $Q_{i}$ as a function of $\bar{n}$ for resonator $B$ might be explained by the saturation of dielectric loss, which is a secondary loss mechanism, accounting for approximately $10 \%$ of the total loss, i.e., $Q_{i}^{-1}$ [see. Fig. 2(c)]. In contrast, $\tau_{\mathrm{ss}}(\bar{n})$ and $Q_{i}(\bar{n})$ for resonator $A$ appear to be correlated [see Fig. 4(b)], and can be fitted by our phenomenological model.

In summary, we characterized grAl superconducting microwave resonators with a kinetic inductance $L_{\text {kinetic }}=$ $2 \mathrm{nH} / \square$, and internal quality factors $Q_{i}>10^{5}$ in the single photon regime, dominated by dissipation due to nonequilibrium QPs. A continuous monitoring of the resonant frequencies shows stochastic QP bursts every $\sim 20 \mathrm{~s}$, followed by an exceptionally long QP relaxation time in the range of seconds, several orders of magnitude longer than in aluminum films $[23,69,73]$, or in Josephson 
junction superinductances [72], presumably explained by single QP localization in regions of lower gap.

Interestingly, despite the disordered nature of the aluminum oxide in-between the aluminum grains in grAl, we measure a similar dielectric loss tangent compared to previously reported values in a variety of pure aluminum superconducting quantum circuits [61]. The coherence properties of grAl resonators are promising for high impedance superconducting quantum circuits and for ultrasensitive KIDs. However, to be able to harvest the full potential of grAl high kinetic inductance films, the density of excess QPs needs to be further reduced, either by elucidating the origin of the QP bursts, which cannot be inferred from our data, or by the use of phonon and QP traps [76].

We are grateful to L. Cardani, M. Vignati, K. Serniak, M. Hays, and M. H. Devoret for insightful discussions, and to L. Radtke, A. Lukashenko, F. Valenti, and P. Winkel for technical support. Facilities use was supported by the KIT Nanostructure Service Laboratory (NSL). Funding was provided by the Alexander von Humboldt foundation in the framework of a Sofja Kovalevskaja award endowed by the German Federal Ministry of Education and Research, and by the Initiative and Networking Fund of the Helmholtz Association, within the Helmholtz Future Project Scalable solid state quantum computing. This work was partially supported by the Ministry of Education and Science of the Russian Federation in the framework of the Program to Increase Competitiveness of the NUST MISIS, Contracts No. K2-2016-063 and No. K2-2017-081.

*ioan.pop@kit.edu

[1] V. E. Manucharyan, J. Koch, L. I. Glazman, and M. H. Devoret, Science 326, 113 (2009).

[2] I. M. Pop, K. Geerlings, G. Catelani, R. J. Schoelkopf, L. I. Glazman, and M. H. Devoret, Nature (London) 508, 369 (2014).

[3] Y.-H. Lin, L. B. Nguyen, N. Grabon, J. San Miguel, N. Pankratova, and V. E. Manucharyan, Phys. Rev. Lett. 120, 150503 (2018).

[4] N. Earnest, S. Chakram, Y. Lu, N. Irons, R. K. Naik, N. Leung, L. Ocola, D. A. Czaplewski, B. Baker, J. Lawrence, J. Koch, and D. I. Schuster, Phys. Rev. Lett. 120, 150504 (2018).

[5] S. Gladchenko, D. Olaya, E. Dupont-Ferrier, B. Douçot, L. B. Ioffe, and M.E. Gershenson, Nat. Phys. 5, 48 (2009).

[6] P. Brooks, A. Kitaev, and J. Preskill, Phys. Rev. A 87, 052306 (2013).

[7] S. Richer, N. Maleeva, S. T. Skacel, I. M. Pop, and D. DiVincenzo, Phys. Rev. B 96, 174520 (2017).

[8] P. Groszkowski, A. Di Paolo, A. L. Grimsmo, A. Blais, D. I. Schuster, A. A. Houck, and J. Koch, arXiv:1708.02886.

[9] A. Petrescu, H. E. Türeci, A. V. Ustinov, and I. M. Pop, arXiv:1712.08630.
[10] O. V. Astafiev, L. B. Ioffe, S. Kafanov, Y. A. Pashkin, K. Y. Arutyunov, D. Shahar, O. Cohen, and J. S. Tsai, Nature (London) 484, 355 (2012).

[11] A. Belkin, M. Belkin, V. Vakaryuk, S. Khlebnikov, and A. Bezryadin, Phys. Rev. X 5, 021023 (2015).

[12] M. T. Bell, W. Zhang, L. B. Ioffe, and M. E. Gershenson, Phys. Rev. Lett. 116, 107002 (2016).

[13] C. Müller, S. Guan, N. Vogt, J. H. Cole, and T. M. Stace, Phys. Rev. Lett. 120, 213602 (2018).

[14] B. H. Eom, P. K. Day, H. G. LeDuc, and J. Zmuidzinas, Nat. Phys. 8, 623 (2012).

[15] M. R. Vissers, R. P. Erickson, H.-S. Ku, L. Vale, X. Wu, G. C. Hilton, and D. P. Pappas, Appl. Phys. Lett. 108, 012601 (2016).

[16] J. Cohen, W. C. Smith, M. H. Devoret, and M. Mirrahimi, Phys. Rev. Lett. 119, 060503 (2017).

[17] S. Puri, S. Boutin, and A. Blais, npj Quantum Inf. 3, 18 (2017).

[18] P. K. Day, H. G. LeDuc, B. A. Mazin, A. Vayonakis, and J. Zmuidzinas, Nature (London) 425, 817 (2003).

[19] J. Aumentado, M. W. Keller, J. M. Martinis, and M. H. Devoret, Phys. Rev. Lett. 92, 066802 (2004).

[20] P. J. de Visser, J. J. A. Baselmans, P. Diener, S. J. C. Yates, A. Endo, and T. M. Klapwijk, Phys. Rev. Lett. 106, 167004 (2011).

[21] V. F. Maisi, S. V. Lotkhov, A. Kemppinen, A. Heimes, J. T. Muhonen, and J. P. Pekola, Phys. Rev. Lett. 111, 147001 (2013).

[22] E. M. Levenson-Falk, F. Kos, R. Vijay, L. Glazman, and I. Siddiqi, Phys. Rev. Lett. 112, 047002 (2014).

[23] C. Wang, Y. Y. Gao, I. M. Pop, U. Vool, C. Axline, T. Brecht, R. W. Heeres, L. Frunzio, M. H. Devoret, G. Catelani, L. I. Glazman, and R. J. Schoelkopf, Nat. Commun. 5, 5836 (2014).

[24] A. Bilmes, S. Zanker, A. Heimes, M. Marthaler, G. Schön, G. Weiss, A. V. Ustinov, and J. Lisenfeld, Phys. Rev. B 96, 064504 (2017).

[25] I. Nsanzineza and B. L. T. Plourde, Phys. Rev. Lett. 113, 117002 (2014).

[26] C. Janvier, L. Tosi, L. Bretheau, ç. ö. Girit, M. Stern, P. Bertet, P. Joyez, D. Vion, D. Esteve, M. F. Goffman, H. Pothier, and C. Urbina, Science 349, 1199 (2015).

[27] S. Gustavsson, F. Yan, G. Catelani, J. Bylander, A. Kamal, J. Birenbaum, D. Hover, D. Rosenberg, G. Samach, A. P. Sears, S. J. Weber, J. L. Yoder, J. Clarke, A. J. Kerman, F. Yoshihara, Y. Nakamura, T. P. Orlando, and W. D. Oliver, Science 354, 1573 (2016).

[28] M. Taupin, I. M. Khaymovich, M. Meschke, A. S. Mel'nikov, and J. P. Pekola, Nat. Commun. 7, 10977 (2016).

[29] K. Serniak, M. Hays, G. de Lange, S. Diamond, S. Shankar, L. D. Burkhart, L. Frunzio, M. Houzet, and M. H. Devoret, arXiv:1803.00476.

[30] A. P. Higginbotham, S. M. Albrecht, G. Kiršanskas, W. Chang, F. Kuemmeth, P. Krogstrup, T. S. Jespersen, J. Nygård, K. Flensberg, and C. M. Marcus, Nat. Phys. 11, 1017 (2015).

[31] D. Aasen, M. Hell, R. V. Mishmash, A. Higginbotham, J. Danon, M. Leijnse, T. S. Jespersen, J. A. Folk, C. M. Marcus, K. Flensberg, and J. Alicea, Phys. Rev. X 6, 031016 (2016). 
[32] U.S. Pracht, N. Bachar, L. Benfatto, G. Deutscher, E. Farber, M. Dressel, and M. Scheffler, Phys. Rev. B 93, 100503(R) (2016).

[33] G. Deutscher, H. Fenichel, M. Gershenson, E. Grünbaum, and Z. Ovadyahu, J. Low Temp. Phys. 10, 231 (1973).

[34] W. Buckel and R. Hilsch, Zeitschrift für Physik 138, 109 (1954).

[35] R. W. Cohen and B. Abeles, Phys. Rev. 168, 444 (1968).

[36] H. Rotzinger, S. T. Skacel, M. Pfirrmann, J. N. Voss, J. Münzberg, S. Probst, P. Bushev, M. P. Weides, A. V. Ustinov, and J.E. Mooij, Supercond. Sci. Technol. 30, 025002 (2017).

[37] V.E. Manucharyan, PhD Dissertation, Yale University, New Haven, CT, 2012.

[38] N. A. Masluk, I. M. Pop, A. Kamal, Z. K. Minev, and M. H. Devoret, Phys. Rev. Lett. 109, 137002 (2012).

[39] M. T. Bell, I. A. Sadovskyy, L. B. Ioffe, A. Y. Kitaev, and M.E. Gershenson, Phys. Rev. Lett. 109, 137003 (2012).

[40] M. R. Vissers, J. Gao, D. S. Wisbey, D. A. Hite, C. C. Tsuei, A. D. Corcoles, M. Steffen, and D. P. Pappas, Appl. Phys. Lett. 97, 232509 (2010).

[41] H. G. Leduc, B. Bumble, P. K. Day, B. H. Eom, J. Gao, S. Golwala, B. A. Mazin, S. McHugh, A. Merrill, D. C. Moore, O. Noroozian, A. D. Turner, and J. Zmuidzinas,Appl. Phys. Lett. 97, 102509 (2010).

[42] L. J. Swenson, P. K. Day, B. H. Eom, H. G. Leduc, N. Llombart, C. M. McKenney, O. Noroozian, and J. Zmuidzinas, J. Appl. Phys. 113, 104501 (2013).

[43] R. Barends, N. Vercruyssen, A. Endo, P. J. de Visser, T. Zijlstra, T. M. Klapwijk, and J. J. A. Baselmans, Appl. Phys. Lett. 97, 033507 (2010).

[44] N. Samkharadze, A. Bruno, P. Scarlino, G. Zheng, D. P. DiVincenzo, L. DiCarlo, and L. M. K. Vandersypen, Phys. Rev. Applied 5, 044004 (2016).

[45] T. M. Hazard, A. Gyenis, A. Di Paolo, A. T. Asfaw, A. S. Lyon, A. Blais, and A. A. Houck, arXiv:1805.00938.

[46] G. J. Grabovskij, L. J. Swenson, O. Buisson, C. Hoffmann, A. Monfardini, and J.-C. Villégier, Appl. Phys. Lett. 93, 134102 (2008).

[47] J. Luomahaara, V. Vesterinen, L. Grönberg, and J. Hassel, Nat. Commun. 5, 4872 (2014).

[48] D. Niepce, J. Burnett, and J. Bylander, arXiv:1802.01723.

[49] F. Levy-Bertrand, T. Klein, T. Grenet, O. Dupré, A. Benoît, A. Bideaud, O. Bourrion, M. Calvo, A. Catalano, A. Gomez, J. Goupy, L. Grünhaupt, U. von Lüpke, N. Maleeva, F. Valenti, I. M. Pop, and A. Monfardini, in preparation (2018).

[50] M. V. Feigelman and L. B. Ioffe, Phys. Rev. Lett. 120, 037004 (2018).

[51] D. Zoepfl, P. R. Muppalla, C. M. F. Schneider, S. Kasemann, S. Partel, and G. Kirchmair, AIP Adv. 7, 085118 (2017).

[52] A. Kou, W. C. Smith, U. Vool, I. M. Pop, K. M. Sliwa, M. Hatridge, L. Frunzio, and M. H. Devoret, Phys. Rev. Applied 9, 064022 (2018).

[53] L. Grünhaupt, U. von Lüpke, D. Gusenkova, S. T. Skacel, N. Maleeva, S. Schlör, A. Bilmes, H. Rotzinger, A. V. Ustinov, M. Weides, and I. M. Pop, Appl. Phys. Lett. 111, 072601 (2017).
[54] See Supplemental Material at http://link.aps.org/ supplemental/10.1103/PhysRevLett.121.117001, which includes Refs. [55-57], for experimental details, further data analysis, and the full phenomenological model.

[55] V. Braginsky, V. Ilchenko, and K. Bagdassarov, Phys. Lett. A 120, 300 (1987).

[56] R. M. Barnett, C. D. Carone, D. E. Groom, T. G. Trippe, C. G. Wohl, B. Armstrong, P. S. Gee, G. S. Wagman, F. James, M. Mangano, K. Mönig, L. Montanet, J. L. Feng, H. Murayama, J. J. Hernández, A. Manohar, M. AguilarBenitez, C. Caso, R. L. Crawford, M. Roos et al., Phys. Rev. D 54, 1 (1996).

[57] S. B. Kaplan, C. C. Chi, D. N. Langenberg, J. J. Chang, S. Jafarey, and D. J. Scalapino, Phys. Rev. B 14, 4854 (1976).

[58] T. Weiß1, B. Küng, E. Dumur, A. K. Feofanov, I. Matei, C. Naud, O. Buisson, F. W. J. Hekking, and W. Guichard, Phys. Rev. B 92, 104508 (2015).

[59] J. Gao, Phd dissertation, California Institute of Technology, 2008.

[60] J. P. Turneaure, J. Halbritter, and H. A. Schwettman, J. Supercond. 4, 341 (1991).

[61] C. Wang, C. Axline, Y. Y. Gao, T. Brecht, Y. Chu, L. Frunzio, M. H. Devoret, and R. J. Schoelkopf, Appl. Phys. Lett. 107, 162601 (2015).

[62] S. J. Weber, K. W. Murch, D. H. Slichter, R. Vijay, and I. Siddiqi, Appl. Phys. Lett. 98, 172510 (2011).

[63] N. Maleeva, L. Grünhaupt, T. Klein, F. Levy-Bertrand, O. Dupré, M. Calvo, F. Valenti, P. Winkel, F. Friedrich, W. Wernsdorfer, A. V. Ustinov, H. Rotzinger, A. Monfardini, M. V. Fistul, and I. M. Pop, arXiv:1802.01859.

[64] S. Hunklinger, W. Arnold, S. Stein, R. Nava, and K. Dransfeld, Phys. Lett. A 42, 253 (1972).

[65] B. Golding, J. E. Graebner, B. I. Halperin, and R. J. Schutz, Phys. Rev. Lett. 30, 223 (1973).

[66] J. Wenner, R. Barends, R. C. Bialczak, Y. Chen, J. Kelly, E. Lucero, M. Mariantoni, A. Megrant, P. J. J. O’Malley, D. Sank, A. Vainsencher, H. Wang, T. C. White, Y. Yin, J. Zhao, A. N. Cleland, and J. M. Martinis, Appl. Phys. Lett. 99, 113513 (2011).

[67] G. Calusine, A. Melville, W. Woods, R. Das, C. Stull, V. Bolkhovsky, D. Braje, D. Hover, D. K. Kim, X. Miloshi, D. Rosenberg, A. Sevi, J. L. Yoder, E. Dauler, and W. D. Oliver. Appl. Phys. Lett. 112, 062601 (2018).

[68] A. D'Addabbo, PhD Dissertation, Université de Grenoble, 2014.

[69] L. J. Swenson, A. Cruciani, A. Benoit, M. Roesch, C. S. Yung, A. Bideaud, and A. Monfardini, Appl. Phys. Lett. 96, 263511 (2010).

[70] D. C. Moore, S. R. Golwala, B. Bumble, B. Cornell, P. K. Day, H. G. LeDuc, and J. Zmuidzinas, Appl. Phys. Lett. 100, 232601 (2012).

[71] L. Cardani, I. Colantoni, A. Cruciani, S. Di Domizio, M. Vignati, F. Bellini, N. Casali, M. G. Castellano, A. Coppolecchia, C. Cosmelli, and C. Tomei, Appl. Phys. Lett. 107, 093508 (2015).

[72] U. Vool, I. M. Pop, K. Sliwa, B. Abdo, C. Wang, T. Brecht, Y. Y. Gao, S. Shankar, M. Hatridge, G. Catelani, M. Mirrahimi, L. Frunzio, R. J. Schoelkopf, L. I. Glazman, and M. H. Devoret, Phys. Rev. Lett. 113, 247001 (2014). 
[73] P. J. de Visser, D. J. Goldie, P. Diener, S. Withington, J. J. A. Baselmans, and T. M. Klapwijk, Phys. Rev. Lett. 112, 047004 (2014).

[74] A. Bespalov, M. Houzet, J. S. Meyer, and Y. V. Nazarov, Phys. Rev. Lett. 117, 117002 (2016).
[75] A. Rothwarf and B. N. Taylor, Phys. Rev. Lett. 19, 27 (1967).

[76] R.-P. Riwar, A. Hosseinkhani, L. D. Burkhart, Y. Y. Gao, R. J. Schoelkopf, L. I. Glazman, and G. Catelani, Phys. Rev. B 94, 104516 (2016). 